\title{
CLIMB Model: Toward a Maturity Assessment Model for Product Development
}

\author{
Monica Rossi ${ }^{(\bowtie)}$ and Sergio Terzi \\ Department of Management, Economics and Industrial Engineering, \\ Politecnico di Milano, Piazza Leonardo da Vinci, 20133 Milan, Italy \\ \{monica.rossi, sergio.terzi\}@polimi.it
}

\begin{abstract}
Product development (PD) becomes crucial for the competitiveness, survival and prosperity of any organization. In order to deliver products successfully, companies can choose between a vast amount of best practices to apply in their innovation processes. However PD processes are still wasteful in practice. With the aim of (i) creating awareness between practitioners on the meaning of PD best practices, (ii) understanding how to measure the maturity in the use of such best practices and in order to (iii) understand the real level of application of these practices, the paper propose CLIMB: a maturity assessment model based on prevalent PD best practices in literature able to measure the maturity of companies in their PD activities. Also the paper proposes the results of an empirical data collection in 2012-2013 within the GeCo Observatory initiative in Italy, which gathered data through face-to-face interviews from more than 100 companies using the CLIMB model. The results is that the tool is effective and that more researches are needed to understand which circumstances lead the choice of certain PD best practices over others.
\end{abstract}

Keywords: Product Development (PD) - Product development assessment · Product development maturity $\cdot$ Assessment tool $\cdot$ Benchmarking $\cdot$ Maturity model $\cdot$ Best practice $\cdot$ Product development best practices $\cdot$ CLIMB model

\section{Introduction: The Need of an Assessment Model for Product Development}

Product development (PD) is the mean by which companies innovate and introduce new product to the marketplace; nowadays PD is becoming more and more crucial for companies competitiveness, prosperity, and survival [1-6]. The success or failure of innovation processes is drastically affected by the choice of engineering and design practices to be implemented during the product development phase. In literature a large number of such engineering and design practices (i.e. tools, methods, techniques) has been explored and studied. Between those, some practices are recognized to foster effectiveness and efficiency of PD and are acknowledged as best practices [7-14]. Both in literature and practice, there is a constant and challenging research of those kinds of best practices and many efforts have already identified a conspicuous number of practices able to lead companies toward successful results [5]. 
However, despite nearly 40 years of scientific research focused on improving PD through the promotion of PD best practices, recent results reveal that these attempts have failed to materialize as expected in practice $[15,16]$. Several gaps are still open. Between those: it is not always clear if practitioners are aware of the meaning of PD best practices [7]; it is not known the level of diffusion of those best practices within industries [5, 7]; and it is in doubt if practitioners are able to identify which practices they could implement in their organizations [7, 13].

These open issues drive the rational of this paper, that aims at covering the existing gaps by providing as a first extent a best practice framework able to create consciousness of what constitutes a best practice in PD (in Sect. 3). The need of a framework is given by the fact that PD is multi-dimensional, and any attempt in literature done to understand the complexity in the variety of PD best practices tries to identify categories of best practices at first. Moreover, basing on this framework, this paper proposes an assessment model (named CLIMB) to be used to assess the maturity of companies in the use of the identified PD best practices (in Sect. 3). The purpose is double: at the first place the aim is to cover the literature gap of understanding the as-is situation in the diffusion of the identified PD best practices in the industrial context. In the second place, as a managerial implication, the authors want to provide companies with a simple and visual assessment tool, to be used both for benchmarking and for self-assessment purposes within companies. PD practitioners are keen to benchmark PD practices because identifying any practice that is able to more efficiently and/or effectively deliver a new product could represent the difference between success and failure [13]. The self-assessment leads to the identification of PD weak areas where to direct improvement efforts. The ultimate purpose of the CLIMB model is to concretely support top management, project managers, and decision makers to identify and select which PD best practices to implement with the hope that companies will manifest and sustain these to expand their PD efforts.

The paper starts from an in depth literature review that, together with several focus groups with experts, have served to build the PD best practice framework and the CLIMB model. Moreover the so-developed maturity assessment model has been used to assess 103 companies in Italy and the results of the level of diffusion of the proposed PD best practices is reported in session 4 of the paper, followed by further thoughts and on-going and future researches in the final session.

\section{State of the Art: Classifications of Best Practices in Product Development}

Any practice whether a technique, a method, a process, or an activity that enables to deliver more efficiency and/or effectiveness than any other manner can be considered as a best practice $[13,17]$. Vice versa, we can define a poor practice.

Product Development is a multidimensional process, constitute of several different but intercorrelated elements across multiple layers and facets. In literature more than 100 PD best practices have been identified, such as the adoption of multifunctional teams, the use of modularization and standardization for parts and components, the use of design for $\mathrm{x}$ techniques, the use of the PLM systems to support the data management through the whole life cycle of a product, for example. 
Given the high number of these practices and the different level they operate, few authors tried the effort of classifying PD best practices across different PD dimensions [1, 10-13], however a unique classification is missing. Some scholars report different grouping of practices at different levels of PD. Under the product development literature field, for example, Barczak et al. (2009) propose 8 different classes [14]: The new PD process, The fuzzy front end (FFE), Portfolio management, Organizing for new product development, Market research tools, Engineering design tools, and Technology \& organizational tools supporting new PD. Similarly to this classification, as a consequence of complementary research activities run within the PDMA (Product Development \& Management Association), another arrangements is suggested as follow [7, 13]: Strategy, Research, Commercialization, Process, Project Climate, Company Culture, and Metrics \& Performance Measurement.

Another attempt of classify principles and practices in product development across dimension, has been given by lean PD literature. The most acknowledged classification is from Morgan and Liker (2006) and consists of the following three areas [1]: Skilled People, Process, and Tools \& Technology.

Despite those different dimensions, both streams acknowledge similar-and sometimes complementary-practices to foster successful product development that inspired the development of the proposed best practice framework at the basis of the CLIMB maturity assessment model.

\section{The CLIMB Maturity Assessment Model}

The CLIMB maturity assessment model aims at covering the above-mentioned gaps (see Sect. 1) by:

(i) Creating awareness on the existing best practices in product development, thanks to the PD best practice framework;

(ii) Providing a useful tool both for scholars - by giving an as - is picture of the current usage and diffusion of PD best practices-and for practitioners - giving them a powerful tool to evaluate their current situation and identifying possible improvements actions based on the benchmarking with what is believed best in literature and eventually with other industrial cases.

The CLIMB maturity assessment model starts from the properly developed PD best practice framework. Then, it builds upon the categories of this framework a maturity model that evaluates 5 different level of accomplishment of the considered categories of best practice. Practically, CLIMB model is composed of (1) a PD Best Practice framework, (2) a questionnaire, (3) a maturity evaluation scale, and (4) a radar chart for a visual representation. All the components are described in the next sections.

\subsection{The PD Best Practice Framework}

Within this study, the authors have identified more than 100 prevalent best practices proposed in literature by different scholars and basing on that, and on a series of focus 
Table 1. PD best practice framework, list and number of best practices (\#BP) for each area

\begin{tabular}{l} 
Area: PEOPLE \\
\hline Sub-Area: Roles \& Collaboration \\
- Cross-functional team \\
- All actors are involved in the project team, even when globally distributed \\
- High flexibility on task execution \\
- There is an overall responsible (PM) with technical background \\
- Full customer involvement in development \\
- Involvement of experienced designers from the earliest stages of the projects \\
\hline Sub-Area: Training \\
- - Formal programs to support multidisciplinary skills development \\
- One-to-one tutoring \\
- KPIs to assess training outcomes
\end{tabular}

\begin{tabular}{l} 
Area: PROCESS \\
\hline Sub-Area: Activities \& Flow \\
- Formal NPD model, properly followed and documented by the various actors \\
involved \\
- Strongly collaborative development process \\
- Complex set of KPIs to measure NPD performance \\
- Frontloading the PD process \\
- Continuous Improvement Initiatives \\
- Many solutions are designed and inferior solutions are progressively \\
discarded when new information becomes available \\
- Complete focus on customer value \\
- Formalized process for analyze competitors (Reverse Engineering) \\
\hline Sub-Area: Decision Making \\
- Lifecycle perspective vision. Consideration of the whole product life phases \\
during PD (10 phases)
\end{tabular}

\section{Area: KNOWLEDGE MANAGEMENT}

Sub-area: KM Process

- Previous knowledge is retrieved by individuals at different PD stages (5 stages)

- Formal overall knowledge management plan

- Main source of knowledge is coming from formal means, such as design rules defined by the company/stakeholders for ensuring the strategic factors are considered in the PD process (12 factors)

- Formal sources of knowledge are continuously update and reviewed (3 formal sources)

- Rely on previous knowledge for PD projects

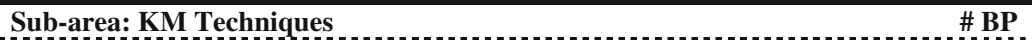

- Structured Tools and techniques formally used to capture, share and reuse knowledge (11 different techniques)

\section{Area: TOOLS}

Sub-Area: Methods

- Formal engineering/design methods (11 methods)

Sub-Area: Computerization \& Software

- Product Development is strongly supported by software platforms (22 softwares) 
groups conducted with experts, they propose a most update framework to collect and categorize PD best practices. The focus groups were constituted by the members of the advisory board of GeCo Observatory - an Italian research initiative created in the frame of the Observatories of the Business School of Politecnico di Milano (http://www. osservatori.net/progettazione_plm). In the specific, 25 practitioners have been consulted together three times during the development and refinement of the framework, and their experience's based suggestions and feedback have been used to develop the final version of the framework.

The framework categorizes 107 PD best practices, through 8 areas, (i) Activities \& Flow, (ii) Decision Making, (iii) Training, (iv) Roles and Collaboration, (v) Knowledge Management Process, (vi) Knowledge Management Techniques, (vii) Methods, (viii) Computerization and Software, respectively grouped into 4 dimensions: Process, People, Knowledge Management and Tools, as summarized in Table (Table 1).

\subsection{The CLIMB Maturity Model}

After developing the proposed best practice framework, the authors developed a maturity model able to associate to each of the practice and category of practice of the framework, a level of accomplishment reached by the respondent.

The maturity model is made of a questionnaire, an evaluation scale made of 5 maturity levels, and a radar chart. The questionnaire is completely based on the proposed PD best practice framework and each of the questions investigates one of the best practices. The number of questions corresponds to the number of the investigated best practices (Table 1). Each question, scored through a 5 points scale, is structured as in the following example (taken from the area Training and Competencies):

Example: How does the company support skills' development?

a. Everyone is personally responsible for developing and maintaining his/her own skills (1)

b. A situation between $a$ and $c$ (3)

c. The company gives training on the job (5)

d. A situation between $c$ and $e(7)$

e. The company promotes multidisciplinary skills with formal programs (i.e. training plans, rotation between project teams) (9)

For each of the more 107 practice five different levels of accomplishment can be selected by the respondent: he/she can choose whether his/her company states at a poor practice level, at a best practice level, or somewhere in between. Those levels can assume a score of 13579 , as reported in the blanks above. The lowest level of accomplishment $(a)$, scored with 1 , corresponds to a poor practice in opposition to the the higher level $(e)$, which corresponds to a best practice, and it is scored with 9. Additionally there are three middle levels, whose intermediate circumstance $(c)$, scored with 5 , is described in order to facilitate the respondent to address his choice. 
A group of one or more questions concurs to describe each of the 8 areas of the framework (Table 1). The score of a single area is calculated as an additive scale (summing the single scores of the questions describing the area) then normalized in $\%$. The following formula defines how the score for each generic area $\left(A_{i}\right)$ is calculated:

$$
\mathrm{a}_{\mathrm{i}}=\frac{\sum_{1}^{\mathrm{m}_{\mathrm{i}}} \mathrm{q}_{\mathrm{ij}}}{8 * \mathrm{~m}_{\mathrm{i}}}
$$

Where:

$a_{i}$ is the score corresponding to i-th area, expressed in $\%$

$i=1 \ldots 8$, is the indicator for the areas

$q_{i j}$ is the score of the answer to the question $\mathrm{j}$, belonging to the $\mathrm{i}$-th area

$j=1 \ldots m_{i}$, is the indicator for the questions, depending on the area the number of questions changes

$m_{i}$, is the number of questions of the $\mathrm{i}$-th area

$8 * m_{i}$ is the maximum score the area can assume in the case the respondent declares to always reach the best practice level-scored with 9-for all the $\mathrm{j}$ practices investigated within the i-th area.

Each of the eight areas expressed in \%, and 5 possible stages of accomplishment of a best practice condition are defined toward the i-th area. The 5 levels are $20 \%$ width intervals in the scale from 0 to $100 \%$ and are namely: Chaos $(0 \%-20 \%)$, Low (21\%$40 \%)$, Intermediate (41\%-60\%), Mature (61\%-80\%), and Best Practice (81\%$100 \%$ ) (Fig. 1). From here the name CLIMB.

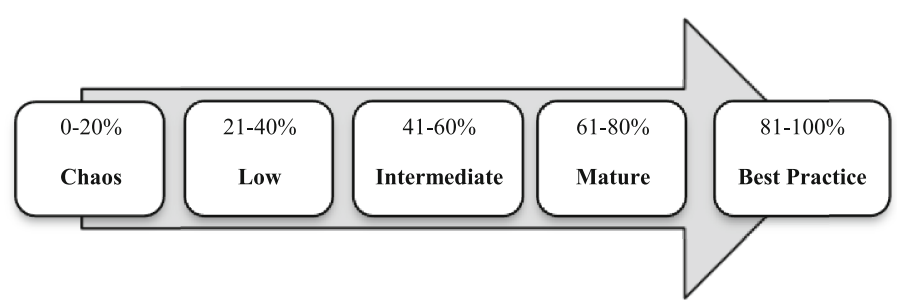

Fig. 1. The 5 maturity levels in the CLIMB model

The level of accomplishment achieved within each of the 8 areas can be then represented in a radar chart (Fig. 2). The radar chart gives an immediate and effective picture of the level of implementation of the considered practices along the eight areas of the framework (Table 1) and displays the positioning of the company within one of the 5 CLIMB stages (Fig. 1). The proposed model could serve as basis for empirical investigations, as the one run in 2012/2013 in Italy and described in the next section. 


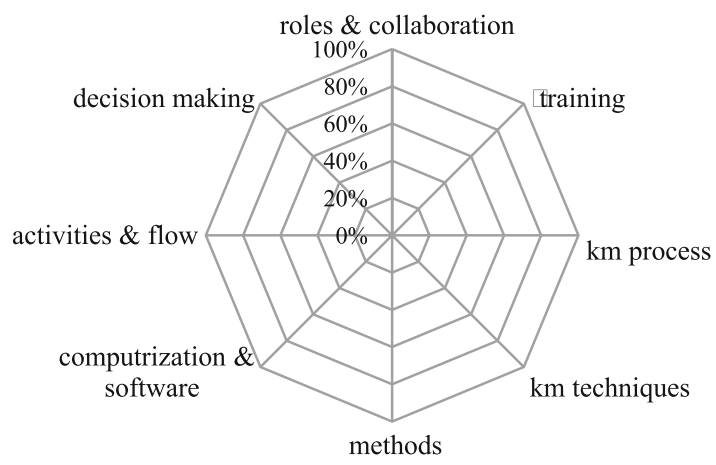

Fig. 2. The radar chart of the CLIMB model

\section{The Diffusion of PD Best Practices in Italy: The Empirical Research of the GeCo Observatory}

In order to evaluate the level of diffusion of the identified PD best practices within industry, and to understand the level of maturity reached by companies in their PD activities, the authors have run an empirical research in Italy, from March 2012 to February 2013. The study has been conducted within the above-mentioned GeCo Observatory initiative on 103 Italian and multinational companies, with at least one product development site in Italy. Each interview involved a project manager, a technical director, and/or a team of engineers working in PD. An average of $2.5 \mathrm{~h}$ have been spent in each company for each face-to-face interview.

The sample is constituted of both small and medium enterprises (SMEs), and big enterprises. Details of the size of the sample are in Table 2. Companies belong to different sectors, grouped into 4: Mechanics, Electrics, Electronics and Other Sectors (such as Fashion, Chemical and Food). Table 3 summarizes the distribution of the sample across the sectors.

Table 2. Sample: size

\begin{tabular}{l|l|l|l}
\hline Size (number of employees) & $\mathrm{N}^{\circ}$ of companies & Class & $\mathrm{N}^{\circ}$ of companies \\
\hline Micro $(<10)$ & 4 & SMEs & 38 \\
\hline Small $(10>$ employees $<50)$ & 13 & & \\
\hline Medium $(50>$ employees $<250)$ & 21 & & \\
\hline Big $(250>$ employees $<1000)$ & 29 & LARGE & 65 \\
\hline Macro $(>1000)$ & 36 & & \\
\hline
\end{tabular}

Figure 3 depicts the radar charts resulting from the empirical research, according respectively to the sectors and the size of the companies belonging to the sample. Despite from one from one could expect, there are not significant differences in 
Table 3. Sample: sector

\begin{tabular}{l|l}
\hline Sector & $\mathrm{N}^{\circ}$ of companies \\
\hline Mechanics & 44 \\
\hline Electrics & 27 \\
\hline Electronics & 18 \\
\hline Other & 14 \\
\hline
\end{tabular}
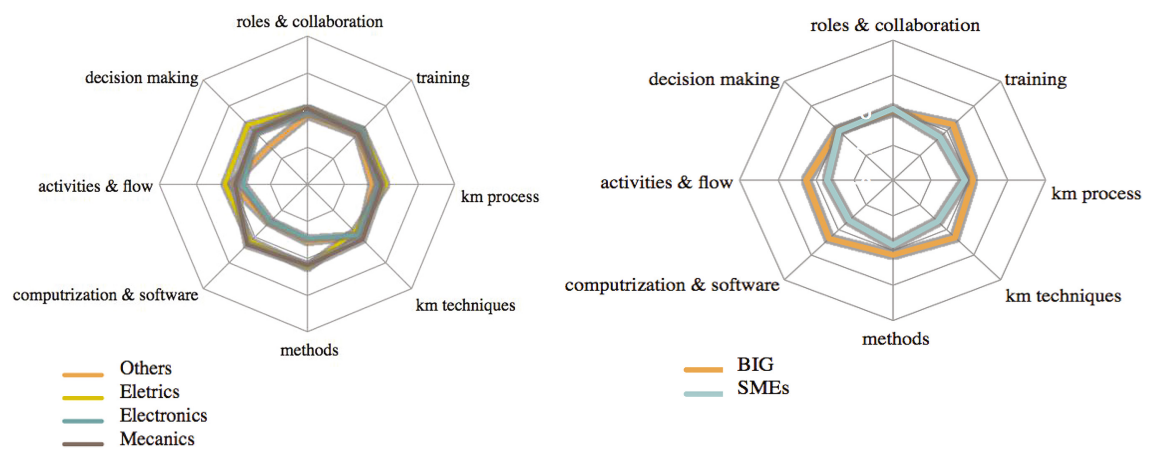

Fig. 3. Current situation of the sample by sector (left) and size (right)

behaviours between BIG and SMEs or between sectors. Areas such as computerization \& software, methods, and knowledge management techniques present the lowest level of maturity within the interviewed sample.

\section{Conclusion and Further Research}

The paper aimed to develop a maturity assessment model, named CLIMB, able to cover the identified gaps of creating awareness on the meaning of PD best practice and creating a way to depict the as-is situation both for scholars and practitioners. The model results effective for the purpose it was created for. Clarity on the meaning of best practice in PD, together with a list and classification of PD best practices are given through the PD best practice framework. The CLIMB model, based on a 5-levels maturity scale, is a powerful tool, useful not only to gather data from companies in the field, but also as a self-assessment tool for mangers. The managerial implications of this piece of work are extremely relevant. From one side respondents become aware of the gap existing between the real level of application of certain best practices in certain areas of PD, compared to ideal practices available to be used. Also thanks to the GeCo Observatory research, companies could benchmark themselves, not only with the best case from literature, but also with the "rest of the world". This is to be considered a first important step toward consciousness on where direct PD improvement efforts.

However, despite the data collected so far don't seems to demonstrate that size or sectors affect the use of best practices in PD, it can't be stated that all the 107 best practices are suitable or ideal to be used in every circumstances and in every company. Logics 
behind the use of proper set of PD best practices driven by contingency variables require higher attention. Further studies should be taken in order to understand if the use of PD best practices is context depended. The GeCo Observatory is going in this direction.

Acknowledgments. This work was partly funded by the European Commission through DIVERSITY (GA_636692) and Manutelligence (GA_636951) Projects, as well as by the GeCo Observatory. The authors wish to acknowledge their gratitude to all the partners for their contributions during the development of concepts presented in this paper.

\section{References}

1. Morgan, J.M., Liker, J.K.: The Toyota Product Development System. Productivity Press, New York (2006)

2. Womack, J.P., Jones, D.T., Roos, D.: The Machine that Changed the World: The Story of Lean Production, Toyota's Secret Weapon in the Global Car Wars that is Revolutionizing World Industry (New Ed.). Simon \& Schuster, London (2007)

3. Womack, J.P., Jones, D.T.: Lean Thinking: Banish Waste and Create Wealth in Your Corporation. Simon \& Schuster, New York (1996)

4. Bayus, B.: Are product life cycles really getting shorter? J. Prod. Innov. Manag. 11(4), 300308 (1994)

5. Griffin, A.: PDMA research on new product development practices: updating trends and benchmarking best practices. J. Prod. Innov. Manag. 14(6), 429-458 (1997)

6. Chesbrough, H., Crowther, A.K.: Beyond high tech: early adopters of open innovation in other industries. R\&D Manag. 36(3), 229-236 (2006)

7. Kahn, K.B., Barczak, G., Nicholas, J., Ledwith, A., Perks, H.: An examination of new product development best practice. J. Prod. Innov. Manag. 29(2), 180-192 (2012)

8. Szulanski, G.: Exploring internal stickiness: impediments to the transfer of best practice within the firm. Strateg. Manag. J. 17(S2), 27-43 (1996)

9. Goodman, P.S.: Critical Issues in Doing Research that Contribute to Theory and Practice. Lexington Books, Lanham (1985)

10. Cooper, R.G., Edgett, S.J., Kleinschmidt, E.J.: Benchmarking best NPD practices-I. Res. Technol. Manag. 47(1), 31 (2004)

11. Cooper, R.G., Edgett, S.J., Kleinschmidt, E.J.: Benchmarking best NPD practices-II. Res. Technol. Manag. 47(3), 50-59 (2004)

12. Cooper, R.G., Edgett, S.J., Kleinschmidt, E.J.: Benchmarking best NPD practices - III. Res. Technol. Manag. 47(6), 43-55 (2004)

13. Barczak, G., Kahn, K.B.: Identifying new product development best practice. Bus. Horiz. 55(3), 293-305 (2012)

14. Barczak, G., Griffin, A., Kahn, K.B.: PERSPECTIVE: trends and drivers of success in NPD practices: results of the 2003 PDMA best practices study. J. Prod. Innov. Manag. 26(1), 3-23 (2009)

15. Flint, D.J.: Compressing new product success-to-success cycle time deep customer value understanding and idea generation. Ind. Mark. Manag. 31, 305-315 (2002)

16. Cooper, R.G.: The invisible success factors in product innovation. J. Prod. Innov. Manag. 16(2), 115-133 (1999)

17. Camp, R.: Benchmarking: The Search for Industry Best Practices that Lead to Superior Performance. ASQ Quality Press, Milwaukee (1989) 